\title{
Turismo y Patrimonio cultural inmaterial: Alternativa de complementariedad para el desarrollo de los territorios rurales
}

\author{
Alonso Rodríguez-Chaves ${ }^{1} \&$ Susan Solís-Rosales ${ }^{2}$
}

1. Encargado de la Cátedra de Historia. Universidad Estatal a Distancia. Costa Rica. arodriguez@uned.ac.cr

2. Encargada de la Cátedra de Turismo Sostenible. Universidad Estatal a Distancia. Costa Rica. ssolis@uned.ac.cr

Recibido: 29 de setiembre, 2015 • Aceptado: 26 de abril, 2016 - Corregido: 16 de agosto, 2016

RESUMEN

(Find the abstracts in English and French at the end of the article)

El nuevo concepto de desarrollo rural generado en las últimas décadas, ha ayudado a redefinir la funcionalidad de los territorios rurales. Esta reconceptualización ha evolucionado en estrecha relación y complementariedad, entre los habitantes y los recursos disponibles en dicho entorno. En ese entramado, el trabajo devela al lector la importancia de la diversificación económica y la consecuente inserción de actividades potencialmente beneficiosa en la vida y quehacer rural. En principio, destaca el aprovechamiento del patrimonio cultural inmaterial como estrategia, para mejorar la competitividad de la actividad turística y, a la vez, para fortalecer las identidades y los rasgos culturales de los colectivos rurales.

Palabras clave: turismo, cultura, patrimonio inmaterial, desarrollo, territorio, rural.

\section{Introducción}

En las últimas décadas del siglo XX, uno de los desafíos de los territorios rurales fue compensar el desequilibrio territorial e infrautilización de los recursos que presentaban esos entornos, asimismo, superar el relativo retraso y aislamiento en que habían quedado. Con ese afán, estos lugares se vieron impulsados a reinventarse y realizar un proceso de

\section{Formato de citación según APA}

Rodríguez-Chaves, A y Solís-Rosales, S. (2016). Turismo y Patrimonio cultural inmaterial: Alternativa de complementariedad para el desarrollo de los territorios rurales. Revista Espiga. Vol. XV, (32), 169-181.

\section{Formato de citación según Chicago}

Rodríguez-Chaves, Alonso y Susan Solís-Rosales. «Turismo y Patrimonio cultural inmaterial: Alternativa de complementariedad para el desarrollo de los territorios rurales». Revista Espiga. XV. n 32 (2016): 169-181. 
revitalización generalizada, la cual se basó en dos principios esenciales: la diversificación y la integración.

Para lograr lo anterior, las comunidades que conformaban esos territorios articularon y generaron acciones integradoras y armoniosas que propiciaron la diversificación económica, el uso de técnicas agrarias respetuosas del ambiente, la competitividad, la mejora de la calidad de vida de los habitantes y el ordenamiento del espacio territorial de conformidad con las lógicas históricas, culturales, naturales, entre otras que les identifican (Delgado y Ramos, 2003).

En general, la solución a esos problemas discurrió dentro de una lógica integral de redefinición que impulsó el desarrollo y consolidación de una cultura empresarial, la cual favoreció en todo sentido, la participación efectiva de los pobladores rurales a nivel familiar o comunal. Igualmente, facilitó la gestión del desarrollo por los mismos actores de los territorios.

Tal fue el alcance de esta perspectiva, que la ruralidad se concibió en el devenir en un sentido amplio, el cual buscaba el bienestar general de la población y la preservación y conservación de todos los recursos que tienen disponible el entorno. Por ende, la visión multifuncional que adquirió el mundo rural incluyó una extensa gama de aspectos en los que el poblador no solo fue visto como el tradicional productor de alimentos sino también como agente potencial para proteger el ambiente y promover el desarrollo sostenible de las comunidades en que habitaba (Vattier, 2005).

Esta tendencia supuso una oportunidad y un contexto favorable para la inserción de nuevas actividades económicas que permitieron a los territorios rurales adquirir mayor reconocimiento, calidad diferenciada, peso en el proceso de comercialización y mejores ventajas comparativas para competir con mayor poder de venta en mercados especiales e internacionales.

Dentro de esa lógica, se incluyó la promoción e implementación de una agricultura equilibrada, competitiva y respetuosa de todo ámbito etnocultural, la cual se complementara con otras actividades sin menoscabar o abandonar los quehaceres productivos tradicionales que se venían practicando desde mucho tiempo atrás. Dicha diversificación conllevó la generación de varias actividades complementarias y relativamente nuevas, las cuales se insertaron con éxito a la vida de los territorios rurales. Este proceso coadyuvó a dinamizar la economía, a elevar el nivel de vida y a obtener otros beneficios de carácter sostenible y perdurable, lo cual permitió mantener a la sociedad económicamente activa.

Con base en esas premisas, este trabajo analiza la inserción de la actividad turística en el marco de la nueva conceptualización de desarrollo rural. Principalmente, invita a reflexionar sobre los elementos potenciales que guarda la actividad económica, y que bien aprovechados por los diferentes agentes que confluyen resultan de suma ventaja para este tipo de entornos. En particular, se refiere a las diferentes expresiones culturales como valores indisolubles e interdependientes que surgen como recursos y estrategias oportunas, atractivas y fácilmente aprovechables en el desarrollo de ofertas turísticas poco diversificadas y exploradas. En general, da importancia a la puesta en valor de estas prácticas y como pueden transcurrir con gran suceso en el ámbito comercial, al ayudar a destinos 
turísticos rezagados a marcar la diferencia y posición privilegiada en los mercados saturados (Ministerio de Cultura. 2009).

Como consecuencia, se propone dar énfasis al patrimonio cultural intangible como aquellas manifestaciones nacidas del sentimiento colectivo y en las cuales descansan significados e información que las sociedades guardan en el imaginario. Con ello se insta a ampliar esquemas habituales de productos turísticos, en los que las comunidades rurales pueden ser realmente competitivas y donde las limitaciones económicas obligan a buscar alternativas cimentadas en el aprovechamiento de capacidades, fortalezas y recursos propios y únicos que posee el entorno rural.

\section{Turismo en la Ruralidad}

El turismo es un fenómeno social en el que las personas se desplazan para conocer nuevos lugares o disfrutar experiencias novedosas que no son parte de su vida cotidiana. Esos viajes tienen una duración que podrían superar las veinticuatro horas, pero inferiores a seis meses, por lo que implica su realización en tiempos no laborales y que incluyan al menos la posibilidad de pernoctar en un lugar tras el final de un día (Quesada, 2010).

Estos movimientos temporales a escala regional, nacional e internacional constituyen una forma de desarrollo económico. Por ende, es necesario señalar que el turismo al ser una actividad socioeconómica, es capaz de establecer encadenamientos con otras actividades. Principalmente, con el sector servicios, donde se genera fuentes de ingresos y empleo en torno a una red de establecimientos que incluyen los hospedajes, restaurantes, tiendas de ropa, equipo deportivo y souvenirs; espacios culturales (museos, centros de baile y clubes nocturnos), las agencias de viaje, las compañías de transporte, los guías, los estudios fotográficos, los mercados de artesanías y las entidades gubernamentales y privadas encargadas de promover la actividad turística (Barahona, 2006).

Para Solís (2015), las tendencias del turismo en la actualidad, consisten en generar o diseñar productos donde las experiencias sean más vivenciales por parte de los turistas. De esta manera, surge un nuevo perfil de turista más exigente y con deseos de adquirir mayor conciencia y conocimientos sobre la preservación y conservación de la naturaleza y de las culturas autóctonas. Para ello, buscan que su desplazamiento geográfico sea optimizado, al visitar lugares donde pueden satisfacer sus expectativas.

En esa perspectiva, no es de extrañar que las particularidades y manifestaciones culturales que presentan las diferentes comunidades sean aprovechadas como ventajas valiosas. En tanto, todo ello es susceptible de influir sobre la valoración que los turistas y compradores de servicios realicen de los productos; pues constituye una cuestión que no debe pasar desapercibida por los nuevos emprendedores del ámbito turístico.

Esta promoción se refuerza con los resultados obtenidos por algunas investigaciones, que reconocen la demanda creciente de productos que encierran en sí mismos, la tradición, costumbres, representaciones colectivas y el saber hacer continuo de los pobladores rurales. De esta manera, el patrimonio cultural intangible puede sorprender como producto favorecedor del turismo, ya que incluye valores y expresiones culturales 
estrictamente ligados a la vida y funcionalidad identitaria de los territorios rurales (Gómez y Caldentey, 2004).

Con ello, los pobladores participan y se benefician de un turismo alternativo sustentable, que además de colaborar con el desarrollo económico local rural, protege y respeta las manifestaciones culturales. A su vez permite al visitante la oportunidad de conocer una cultura y disfrutar de una actividad lúdica pedagógica, capaz de generar experiencias únicas; pues se percibe como estrategia donde logra el nexo y cohesión común con elementos culturales de particular especificidad de las comunidades rurales.

\section{El potencial del patrimonio cultural en el desarrollo turístico rural}

El patrimonio cultural es una construcción social, que enlaza el ayer, el hoy y el mañana. Un bien patrimonial tiene su origen en el pasado, pero gracias al paso intergeneracional, logra llegar hasta nuestros días, donde puede ser re-elaborado o re-significado. De este modo, mientras guarde sentido y gran significado para el grupo social que lo recibe, lo porta, lo cuida y lo transmite a las futuras generaciones, seguirá siendo algo compartido (UNESCO, 2012).

En virtud de lo anterior, el patrimonio puede comprender tanto objetos como saberes plasmados en técnicas, prácticas, creencias y rituales. En otras palabras, el patrimonio engloba un conjunto de pertenencias tangibles e intangibles que un determinado grupo cultural considera como valiosas, por ser parte, de una herencia compartida con el resto de la comunidad y que otorga un sentido de identidad a quienes lo poseen.

En la delimitación de lo patrimonial, se debe tomar en cuenta que el dinamismo se presenta como un factor esencial, pues esto evitará caer en la simplificación y en el error de reducir el patrimonio al pasado y a la conservación de objetos o costumbres casi intactas, ya inutilizadas y sin ninguna función social. En razón, un enfoque equivocado sería suponer que el patrimonio cultural comprende solo los elementos que son exclusivos de un pueblo o grupo social que habita un territorio rural en particular (UNESCO, 2012).

Para comprender mejor lo indicado, basta recordar que una constante en la raza humana desde su existencia ha sido la movilización y desplazamiento por diferentes territorios, lo que ha implicado diferentes formas de contacto de índole intercultural. De igual manera, ocurre cuando un turista regresa a su hogar después de haber realizado un viaje, retoma otras experiencias y visiones de lo que es el mundo y la vida, por ende, algunas de ellas serán asimiladas con adaptaciones al contexto cultural en que vive.

Por consiguiente, el patrimonio hay que vincularlo a la herencia de hechos culturales, que un grupo considera como bienes propios, que son compartidos por una comunidad y actúan como referentes identitarios de la misma. El reconocimiento por parte de la colectividad que produce un determinado bien cultural y su valoración como tal, implica un proceso - muchas veces inconsciente- de jerarquización y selección entre una amplia variedad de hechos culturales (UNESCO, 2012). 
En consecuencia, el patrimonio cultural nos remite a nuestras raíces, a la búsqueda del legado ancestral. Si bien su origen se halla en el pasado, no todo lo que procede de allí se mantiene, pues gracias a un proceso de resemantización y resemiotización se producen transformaciones totales o parciales en uno o varios componentes de un hecho cultural.

Es decir, la herencia está sujeta a renovaciones y resignificaciones, lo que se evidencia en cambios en la función social, la forma o el estilo, el simbolismo, los elementos materiales que intervienen en su elaboración, entre otros. De tal manera, que algunos elementos se les atribuye distinto sentido y si no se refrescan se tienden a perder para siempre.

En general, todo bien patrimonial es un hecho cultural, sin embargo, no todo hecho cultural tiene valor patrimonial. La explicación de esta afirmación radica en que la cultura de todo grupo social es sígnica, es decir, posee una serie de significados particulares al grupo social o comunidad que los produce y usa. A esto debemos agregar que un mismo hecho cultural puede tener distintos significados, ya que éstos son asignados por un determinado grupo social, con base en elementos que le dan sentido.

En suma, el patrimonio cultural se puede dividir en patrimonio material o tangible y patrimonio inmaterial o intangible, aunque en la realidad concreta hay una permanente interrelación y reciprocidad entre ambos y el ambiente en que se construyeron. En la realidad concreta es difícil marcar el límite entre lo material y lo intangible. La distinción entre ambos tipos de bienes se realiza con fines taxonómicos a la hora de ordenar y clasificar datos culturales. Un ejemplo de patrimonio material o tangible puede ser todo aquello que se encuentra legado en soporte físico y patrimonio inmaterial o intangible aquello que se encuentra legado a través de significados culturales (Ministerio de Cultura, 2009).

En el patrimonio material a su vez se distinguen dos tipos diferentes de bienes: los "muebles", denominados así debido a su carácter de objetos móviles. Por consiguiente, corresponde a los objetos artísticos, etnográficos y documentos visuales de valor histórico escritos o gráficos y, los "inmuebles", que corresponden a aquellos bienes que no cambian de lugar donde fueron erigidos, como los bienes arquitectónicos y algunos arqueológicos(Ministerio de Cultura, 2009).

Por su parte, el patrimonio intangible, se cifra en la oralidad y engloba los saberes y conocimientos, las prácticas sociales, las técnicas con que se elaboran ciertos objetos. Según la Convención para la Salvaguarda del Patrimonio Cultural Inmaterial de la Organización de las Naciones Unidas para la Educación y la Cultura (UNESCO) celebrada en el año 2003 se anota la siguiente definición:

El patrimonio cultural no se limita a monumentos y colecciones de objetos, sino que comprende también tradiciones o expresiones vivas heredadas de nuestros antepasados y transmitidas a nuestros descendientes, como tradiciones orales, artes del espectáculo, usos sociales, rituales, actos festivos, conocimientos y prácticas relativos a la naturaleza y el universo, y saberes y técnicas vinculados a la artesanía tradicional (UNESCO, 2012, p12).

La UNESCO de igual forma menciona las siguientes características que permitirán orientar de mejor manera, la comprensión del concepto y que demuestra la evolución del mismo en el tiempo: 


\section{Caracterización del patrimonio cultural}
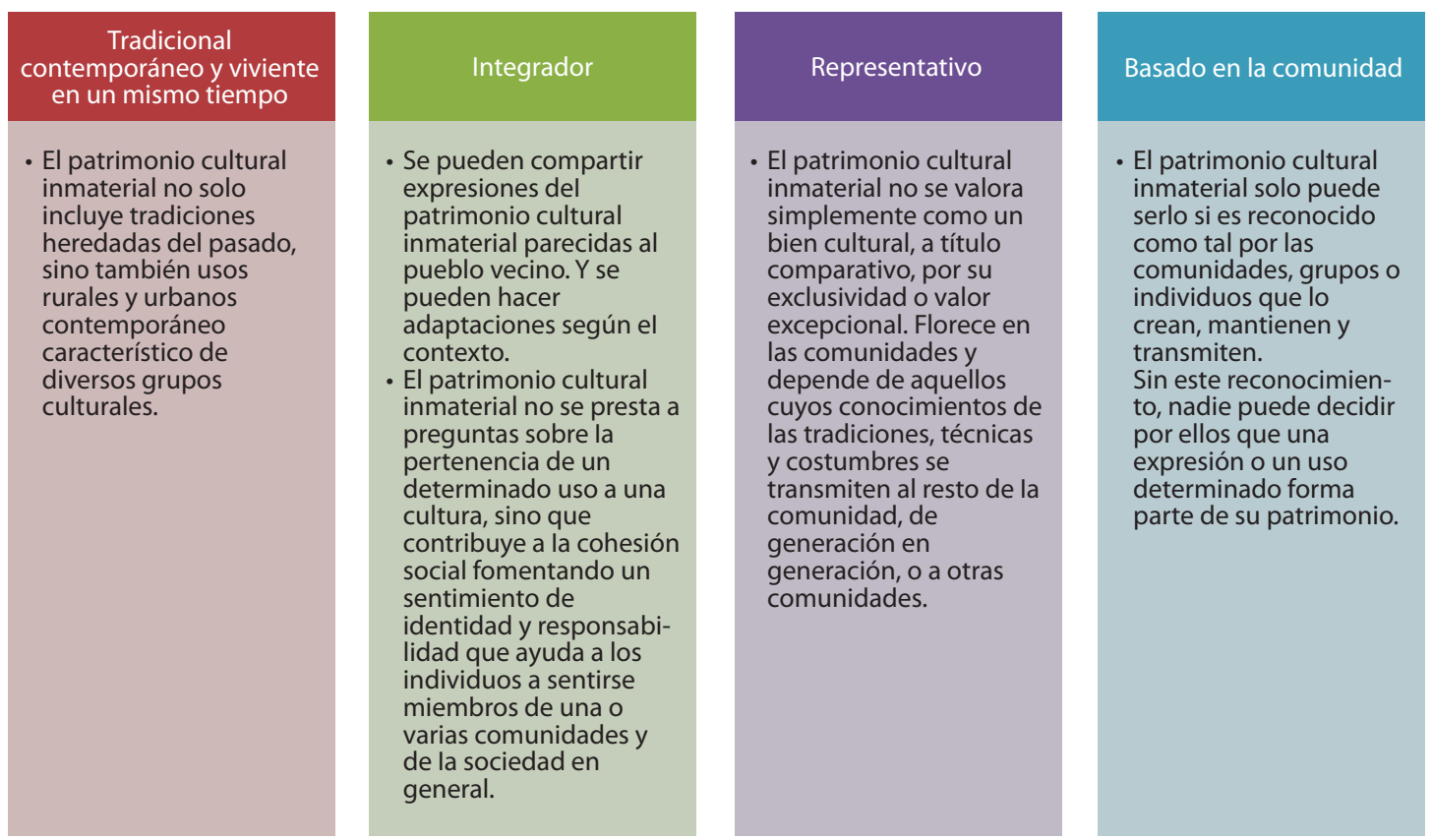

Fuente: Elaboración propia a partir de Patrimonio Inmaterial de Organización de las Naciones Unidas para la Educación y la Cultura (UNESCO), 2012.

La caracterización indicada permite tener una mayor claridad de lo que cada territorio puede generar en materia de patrimonio cultural inmaterial. Asimismo, muestra que no es necesario que las manifestaciones culturales correspondan a un pasado muy antiguo o más reciente; lo importante es que determinen, signifiquen, identifiquen y representen a uno sino a varios colectivos vecinos.

Entre tanto, la definición mostrada es enfática con respecto al valor que le pueda dar la comunidad o colectivo al patrimonio cultural, ya que nadie puede decidir que será representativo para ellos o ellas. Es el grupo mismo, el cual tiene que realizar esa determinación, siendo esto un aspecto relevante en la incursión de las diversas actividades que se deseen desarrollar. Por ejemplo: en la actividad turística se requiere una planificación integradora, no solo desde el punto de vista operativo sino conceptual desde la visión del colectivo que compartirá su cultura o identidad.

En la actividad turística se ha identificado una tipología según las motivaciones de los visitantes en aras de comprender y ofrecer los servicios según las necesidades privativas de cada perfil de turista. En el caso particular de la temática del patrimonio cultural se puede mencionar las definiciones de las tipologías:

A. Turismo étnico: Mc Intosh, Goeldner y Ritchie (2002) indican que son viajes con el objetivo de observar las expresiones culturales y el estilo de vida de los pueblos con 
características exóticas. Y que las actividades usuales serían visitar hogares nativos, participar de bailes, ceremonias o rituales religiosos.

B. Turismo cultural: según Quesada (2010) este tiene una mayor concepción, en tanto, las actividades que realiza se podrían ver desde el punto de vista del arte en su sentido amplio. Tal es el caso, de visitar sitios históricos de culturas ancestrales como los egipcios, griegos, mayas, entre otras. También podría interesarse por conocer alguna cultura particular, pero quizás el nivel de inserción en el grupo étnico no sea de mayor profundidad como la tipología mencionada anteriormente.

Ahora bien, a la hora de incursionar el patrimonio turístico con el sistema turístico la Organización Mundial del Turismo (OMT) hace el llamado que esta ecuación debe desarrollarse con cautela, ya que podría afectarse y alterarse la razón de ser de un colectivo o comunidad debido al contexto globalizado en que se encuentra la sociedad y además de las diversas visiones que se tiene del mundo.

Al referirse al problema del control cultural, el antropólogo mexicano Guillermo Bonfil Batalla (2004) distinguió la presencia de elementos propios y ajenos en la cultura de cualquier grupo social. Su propuesta es una herramienta de gran utilidad para resolver la cuestión de cuál parte de la cultura o de sus manifestaciones pueden considerarse patrimoniales a partir del reconocimiento de lo propio y lo ajeno.

El ejercicio puede parecer muy simple y realizarse con cualquier componente de la cultura, así descubrir o reconocer la complejidad que acarrea. Por ejemplo, se tiene expresiones de la cultura que son propias, con un carácter de singularidad que las distingue de otros hechos culturales semejantes de otros pueblos.

Lo anterior conduce a señalar que hay elementos que son compartidos en diferentes culturas, ya sea porque tienen un origen común o porque se dio un proceso de apropiación y de intercambio de algunos elementos. Tal es el caso, de varias expresiones culturales de origen europeo o latinoamericano, que producto del proceso de colonización se incorporaron a las culturas nacionales. Esto ayuda a entender la dinámica representada en la variante gastronómica, en la cual los europeos aportaron el trigo y las culturas autóctonas el maíz.

En el texto incluye el término conservación en un sentido amplio, en consecuencia engloba otras acciones muy similares como la preservación y el mantenimiento. Sin embargo, la palabra conservación tiene una mayor carga semántica, que integra el carácter dinámico que posee el patrimonio cultural.

Ningún bien cultural debe permanecer invisibilizado, sino que casi cualquier bien puede ser objeto de alguna actividad en pro de su implementación cotidiano; como en el caso de las tradiciones populares y otros bienes intangibles que pueden ser dinamizados o revitalizados a través del turismo y de las actividades conexas.

Es importante mencionar, que no vale de nada conservar el patrimonio si no contamos con una propuesta efectiva de gestión de este. De esta manera, no tiene sentido preservar por preservar un bien cultural, sino que el reto es transformar ese bien intangible en un recurso cultural, que esté al servicio del desarrollo, mediante proyectos educativos, científicos o turísticos. 
La actividad turística puede ser un arma de doble filo en el logro de esta meta de proteger y conservar; pues por un lado, la visita de numerosas personas a un sitio o comunidad, algo muy común en el turismo de masas, en algún momento llega a desequilibrar y perturbar el ambiente natural y cultural.

No obstante, la visita equilibrada y basada en la educación, puede ser una colaboradora en la tarea de proteger el patrimonio. Al respecto Guereña y Calderón (2005: 131) indican los siguientes aspectos:

A. Autenticidad del hecho cultural: debe ser algo existente y nunca inventado. Si se quiere mostrar una actividad cotidiana, es preferible adaptar el horario de la visita al momento en que se produce esa actividad, en lugar de recrearla.

B. Autenticidad de las personas: el protagonismo lo debe siempre tener la persona local, que es quien tiene el conocimiento y el orgullo de lo que hace. El papel del guía externo debe ser el de facilitador.

C. Tradición como proceso: es interesante presentar la evolución de lo que se observa, preguntarse cómo ha llegado a ser lo que es hoy. Entender la cultura como algo vivo y dinámico, que va evolucionando.

D. Información correcta: Se debe dominar la historia local y el origen de lo que se presenta. Ser fiel a la tradición y a las costumbres, sin adulterarlas.

E. Respeto al ambiente: No ir en contra de principios ambientales.

Entre las expresiones culturales que se pueden desarrollar en la oferta turística de Costa Rica se mencionan algunos ejemplos a continuación:

\section{Expresión cultural}

Festivales

\section{Ejemplo}

Festival Académico Cultural Guanacastequidad organizado por Ministerio de Educación Pública (MEP), Universidad Estatal a Distancia (UNED) y diversos actores locales.

\begin{tabular}{ll}
\hline Ferias agroalimentarias & Feria del Café en Frailes de Desamparados (Costa Rica). \\
\hline Mascaradas & Máscaras de Heredia y Escazú (Costa Rica). \\
\hline Tradiciones alimenticias & $\begin{array}{l}\text { Alimentos que se han dejado de consumir pero han sido tradición cultu- } \\
\text { ral, principalmente con poblaciones indígenas: chicasquil (Cnidoscolus } \\
\text { aconitifolius), helecho rabo de mico (Phlebodium aureum), cohombro } \\
\text { (Cucumus flexuosus), oreja de palo (Ganoderma australe), entre otros. }\end{array}$ \\
\hline Artesanías locales & $\begin{array}{l}\text { Artesanías de San Vicente y Guaitil con origen étnico Chorotega, los } \\
\text { cuales se producen en la provincia de Guanacaste de Costa Rica. }\end{array}$ \\
\hline Tradición oral & Leyendas o cuentos tradicionales. \\
\hline
\end{tabular}

Fuente: Elaboración propia 
Los anteriores son algunos ejemplos que se pueden encontrar en las comunidades costarricenses. No obstante, su puesta en valor no solo sirve para su apropiación y reforzamiento cultural, sino también para su incorporación en la actividad turística, la cual dependerá del interés e identificación que muestre el colectivo local. De lo contrario, se entraría en una banalización y mera comercialización del elemento cultural (García, 1990).

En ese contexto, las organizaciones no gubernamentales (ONG) y cooperantes internacionales conjuntamente con las organizaciones de base comunal inician un proceso de capacitación y profundización para incentivar el desarrollo de ofertas turísticas, que preserven y conserven los valores naturales y culturales que poseen las diferentes comunidades y colectivos rurales.

En el caso de Costa Rica, si bien posicionó su marca país a nivel mundial por sus valores naturales, en los últimos años ha promovido también, los elementos identitarios como parte del producto turístico potencial. Los esfuerzos en la materia se consideran en las políticas públicas representadas en el Plan Nacional de Turismo en Costa Rica a través del Certificado de Sostenibilidad Turística (CST) donde se incentiva a empresarios turísticos a exaltar los aspectos culturales de índole nacional y local, o según donde se ubique el establecimiento turístico.

Además, este instrumento promueve la sensibilización por parte de los turistas que visitan dichos sitios para que se acerquen a la cultura que están visitando y se lleven experiencias únicas. El hecho se puede evidenciar desde el surgimiento del Turismo Rural Comunitario (TRC), que promueve dichos valores, y que, con la recién adhesión de Costa Rica a la Organización Internacional de Turismo Social (OTIS), se espera fortalezca la política de Estado para ampliar las posibilidades de aprovechar y disfrutar de la cultura, no solo para el turista internacional, sino también para el nacional, al permitir mayores incentivos y subsidios para sus desplazamientos tal como ocurre en países que han demostrado por décadas éxito al respecto, a saber: Brasil, México, Chile, Colombia y Argentina, entre otros.

Dentro de esa nueva conceptualización, el turismo se asoma al ámbito rural como una actividad vigorosa, dinámica y sumamente prometedora; considerando que no solo ayuda a modernizar y diversificar, sino a complementar la economía tradicional. No es casualidad entonces, que sea identificada y señalada esta y otras actividades que van surgiendo como "motores de crecimiento", debido a los efectos directos que logran causar en el ámbito rural; pues contribuyen a aumentar el ingreso, reducir marginalmente la pobreza a través de nuevas oportunidades para el crecimiento (Gutiérrez, 2006).

Ante ese escenario que reparan y exigen los tiempos recientes, resulta importante continuar profundizando en la actividad turística. En principio, innovando con nuevos componentes culturales como los mencionados, los cuales pueden consolidar las ventajas de acceso de los territorios rurales a los mercados exigentes y potencialmente beneficiosos. Cabe mencionar que las estrategias comerciales, en los últimos años, reconocen el valor especial de la ruralidad, en especial, las personas y sus especificidades culturales que expresan a través de productos (Caldentey y Gómez, 1998).

Es importante señalar, como en una desmesurada liberalización comercial o coyuntura de crisis internacional, la promoción de estas respuestas innovadoras pueden 
ayudar a solventar o afrontar de mejor manera, las vicisitudes posibles que han de venir. También aprovechables como "valor agregado" para hacer frente a la eventual entrada y competencia de otros mercados que participan dentro de la actividad turística (Damiani, 2005 y Ulate, 2005).

De ser así, compromete indirectamente a los emprendedores de comunidades rurales a desarrollar y perfeccionar los estándares de calidad relacionados con la actividad turística; que a la postre, se pueden convertir en verdaderas ventajas e incidir positivamente en el desarrollo rural (Banco Interamericano de Desarrollo. Departamento de Desarrollo Sostenible, (2009).

Según lo anterior se puede mencionar el caso que en el año 2001 la Asociación para el Desarrollo Sostenible de San José Rural (ADESSARU) en aras de generar un ingreso económico para promover actividades ambientales y por ende dinamización local, construye un albergue de turismo rural comunitario tal como el que se produce con Nacientes Palmichal en la comunidad de Palmichal de Acosta ${ }^{1}$. Tal y como apunta Guereña (2005) en este tipo de emprendimientos turísticos tan particulares se "desarrollan un concepto propio de calidad, que se refleja en el servicio personalizado de los empleados y los asociados, un servicio cálido, respetuoso y amigable, que hace sentir al visitante a gusto, como si estuviese visitando un pariente lejano" (p.82)

\section{Conclusión}

Las últimas décadas del siglo XX significaron un exitoso periodo de políticas de desarrollo rural con el fin de mejorar todos los aspectos de la vida de esos territorios. De este modo, la reconceptualización de los espacios rurales evoluciona en una relación estrecha entre los intereses de los pobladores por mejorar la producción tradicional y la preservación y conservación de los recursos propios del entorno rural.

Al margen de la riqueza productiva tradicional, la diversificación se presenta como una gran oportunidad para contribuir a la gestión y producción de las comunidades. Así aparecen actividades complementarias que ayudan a dinamizar la vida y la economía de los territorios rurales. No es casualidad, que algunos refieren y relacionen esas nuevas actividades como "la fuerza de la diferencia", pues bien aprovechadas llegan a ser vitales en territorios rurales que urgen de alternativas económicas para sobrevivir y competir mejor en los actuales mercados (Llorente, 2001).

En ese sentido, esta ecuación incuestionable se mira como fortaleza y que bien aprovechada colabora en la revitalización rural. Conforme a lo expuesto, a través de acciones enfocadas a escala local pueden encaminar a las comunidades rurales desfavorecidas a una situación de mejora en calidad de vida; de bienestar socioeconómico; concienciación

1. Para saber más sobre la dinamización local en materia de turismo rural comunitario se recomienda visitar el http://www.nacientespalmichal.com/es/. Este es un emprendimiento turístico que se desarrolla en comunidad de Palmichal de Acosta, Costa Rica. 
y mentalización colectiva frente las problemáticas del mundo rural; sostenibilidad y perdurabilidad de los valores naturales y culturales.

En esa dirección, la aparición del turismo como actividad económica ayuda a consolidar el planteamiento que han venido gestando las comunidades rurales a través de proyectos que respeten las formas de vida tradicional, da importancia a preservar y conservar los recursos e impulsar el desarrollo económico como elementos sostenibles e integrales (UNESCO, 2007).

No obstante, la actividad turística sigue siendo potencial pese el gran auge que ha tenido, ya que hay productos culturales que no se aprovechan adecuadamente por las comunidades rurales. Sin duda, estos argumentos se justifican cuando se sabe que existe abundante y valioso patrimonio cultural, conformado por una amplia gama de expresiones y manifestaciones, que en el tiempo han venido a representar símbolos de identidad para los pobladores de los entornos rurales.

Dentro de esas expresiones transmitidas de una generación a otra encontramos una serie de cosmovisiones, lenguajes, formas de relación, valores estructurados, tradiciones, costumbres y saberes particulares que dan como resultado un producto cultural intangible particular, que aprovechadas responsablemente ampliarían la oferta turística, la competitividad y mayor valor en el mercado (Arias y Vives, 2005).

En general, toda ampliación de la oferta turística debe ser cuidadosamente revisada, convenida e implementada con el objetivo de no violentar, masificar o trastornar la forma de vida y la cultura rural por el mero hecho de la comercialización. Queda demostrado que es posible generar un desarrollo con esta vinculación: turismo y patrimonio cultural inmaterial, no obstante, debe ser primero apropiada y aprobada por las comunidades, tanto para que sea legítima como efectiva.

\section{ABSTRACT}

Turism and cultural nonmaterial patrimony: alterative of complimentary for the development for rural territories

The new concept of rural development created in the last decades has helped to redefine the usefulness of rural territories. This reconceptualization has evolved linked and in relation to the residents and the available resources in the surroundings. In this framework, the paper depicts the importance of the economic diversity and the resulting insertion of potentially beneficial activities in the life of the rural job. At the beginning, it highlights the exploitation of the cultural nonmaterial patrimony as strategy to improve the competitively of the touristic activity, and at the same time, to strengthen the identities and cultural characteristics of the rural groups.

Key words: tourism, culture, nonmaterial patrimony, development, territory, rural 


\section{RÉSUMÉ}

\section{Tourisme et Patrimoine culturel immatériel: alternative de complémentarité pour le développement des territoires ruraux}

Le nouveau concept de développement rural, suscité dans les dernières décennies, a permis de redéfinir la fonctionnalité des territoires ruraux. Cette reconceptualisation a évoluée en étroite collaboration et complémentarité, entre les habitants et les ressources disponibles de cet environnement. Dans ce cadre, le travail révèle au lecteur l'importance de la diversification économique et par conséquence l'inclusion d'activités potentiellement bénéfiques dans la vie et la quotidienneté rurale. Initialement, il est remarqué l'exploitation du patrimoine culturel immatériel comme stratégie pour améliorer la compétitivité de l'activité touristique, ainsi que pour renforcer les identités et les traits culturels des sociétés rurales.

Mots-clés: Tourisme, culture, patrimoine immatériel, développement, territoire, rural.

\section{Referencias bibliográficas}

Arias, R. y Vives, I. (2005). Doka Estate: Historia y arquitectura de una hacienda cafetalera. En: Revista Patrimonio. Centro de Investigación y Conservación del Patrimonio Cultural. Ministerio de Cultura, Juventud y Deportes. Año 5; (5), San José.

Banco Interamericano de Desarrollo. (2009). Departamento de Desarrollo Sostenible. Agricultura y Desarrollo Sostenible. Recuperado de: http://www.iadb.org/SDS/ENV/ site_47_s.htm

Bonfil, G. (2004). Patrimonio Cultural Inmaterial. En: Diálogos en la acción, primera etapa. Recuperado de: http://www.ilam.org/ILAMDOC/sobi/Guillermo\%20Bonfil_Pensarnuestracultura-Pat.pdf

Caldentey, A. y Gómez, A. (2001). Productos típicos y denominaciones: de la tutela a la divergencia. En: IV Coloquio hispano-portugués de estudios rurales. Dpto. de Economía, Sociología y Política Agrarias. Universidad de Córdoba. Santiago de Compostela.

Caldentey, P. y Gómez, A. (1998). Estudio para la implantación de signos de calidad en productos agroalimentarios de la Sierra Norte de Sevilla (doc. mimeo.)

Damiani, O. (2005). Adversidad y cambio: estrategias exitosas de pequeños productores de café en Centroamérica. 1 edición. San José: Serie de publicaciones RUTA.

Delgado, M. (2001).Análisis de los efectos de la nueva política rural europea: una aplicación al caso andaluz. Tesis de Doctorado. Departamento de Economía Agrícola, Escuela Superior de Ingenieros Agrónomos y Montes, Universidad de Córdoba. Córdoba, España.

Delgado, M. y Ramos, E. (2003). Understanding the evolution of the European Rural Policy: A methodological approach(Monografía de Investigación). Universidad de Córdoba, Departamento de Economía Agrícola, Escuela Superior de Ingenieros Agrónomos y Montes. Córdoba, España.

García, N (1990). Culturas híbridas. Estrategias para entrar y salir de la modernidad. México: Editorial Grijalbo.

Guereña y Calderón (2005). Turismo Rural Comunitario en Costa Rica. La experiencia del Programa de Pequeñas Donaciones y sus Grupos Socios. San José: Programa de las Naciones Unidas para el Desarrollo (PNUD). 
Gutiérrez, T. (30 de setiembre, 2006). Exigen reglamento sobre indicaciones geográficas. En: La Prensa Libre. San José.

Hernández, J. (2011). Los caminos del Patrimonio. Rutas turísticas e itinerarios culturales. En: Revista Pasos. Vol.9. (2): 225-236. Recuperado de: http://pasosonline.org/ articulos/469-los-caminos-del-patrimonio-rutas-turisticas-e-itinerarios-culturales

Llorente, M. (2001). La fuerza de la diferencia: la denominación de origen, un instrumento para el desarrollo. Huesca: Ediciones La Val de Onsera.

McIntosh, R; Goeldner, C y Ritchie, J. (2002).Turismo Planeación, Administración y Perspectivas. México: Editorial Limusa.

Ministerio de Cultura. (2009). Conclusiones de las Jornadas sobre Patrimonio Inmaterial. Teruel: España.

Nacientes Palmichal. (2016). Acosta, Costa Rica. Recuperado de: http://www.nacientespalmichal.com/es/

Organización Mundial del Turismo (OMT) (2013). Resumen de los resultados: Estudio sobre el turismo y el patrimonio cultural inmaterial. Recuperado de: file:///D:/ art $\%$ C3 $\%$ ADculo $\% 20$ edici $\% \mathrm{C} 3 \% \mathrm{~B} 3 \mathrm{n} \% 20$ especial/summarytourismandichstudy120512esprint.pdf

Quesada, R. (2010). Elementos de Turismo. (2 ed.) San José: EUNED.

Solís, S. (2015). Tendencias del desarrollo turístico mundial. Manuscrito en preparación.

Organización de las Naciones Unidas para la Educación y la Cultura (UNESCO). (2012). Patrimonio Inmaterial. Recuperado de: http://www.unesco.org/culture/ich/index. php?lg=es\&pg $=00002$

Organización de las Naciones Unidas para la Educación y la Cultura (UNESCO).(2007). Un Museo Sostenible: museo y comunidad en la preservación activa del patrimonio. San José.

Organización de las Naciones Unidas para la Educación y la Cultura (UNESCO).(2003). Convención para la Salvaguarda del Patrimonio Cultural Inmaterial.

Vattier, F. (2005). Las nuevas orientaciones normativas de la PAC y el desarrollo rural. La Rioja. 
